\title{
Coronary artery_steal via large coronary artery to bronchial artery anastomosis successfully treated by operation
}

\author{
M G ST JOHN SUTTON, G A H MILLER, I H KERR, T A TRAILL \\ From the Brompton Hospital, Fulham Road, London
}

SUMMARY We report a patient with a large coronary artery to bronchial artery anastomosis causing angina by coronary steal. Angina was refractory to medical treatment, but successfully relieved by surgical ligation of the anastomosis.

Extracardiac coronary anastomoses are rare and have received little attention since the initial necropsy description in $1803 . .^{1}$ Since the introduction of angiography small anastomoses have been shown between the coronary and bronchial arterial circulations in normal subjects, ${ }^{2}$ which have little, if any, haemodynamic significance. We report a patient with a large coronary artery to bronchial artery anastomosis and bronchiectasis in whom shunting of blood from the coronary circulation resulted in angina, that is, a coronary steal syndrome. His angina was refractory to medical treatment but was relieved by surgical ligation of the anastomosis and resection of the bronchiectatic left lower lobe.

\section{Case history}

A 56-year-old man was admitted for investigation of recurrent severe angina pectoris which had been present for one year; in addition, there had been two episodes of prolonged chest pain occurring at rest and associated with sweating. $\mathrm{He}$ gave a history of spontaneous pneumothorax resulting from a ruptured emphysematous bulla, and for 15 years he had suffered from bronchial asthma which was treated with bronchodilators.

Examination of the cardiovascular system was normal. Respiratory excursion was reduced, and there was an inspiratory and expiratory wheeze over the middle and lower zones on both sides, and crackles over the left lower and middle zones.

Examination of the cardiovascular system was normal. Respiratory excursion was reduced, and there was an inspiratory and expiratory wheeze over the middle and lower zones on both sides, and crackles over the left lower and middle zones:

Electrocardiogram showed sinus rhythm, a normal $Q R S$ vector, and flat $T$ waves in leads III, aVF, and V5-6. During the exercise electrocardiogram, a heart rate of 146 beats per minute was attained, but there were no further electrocardiographic abnormalities. Chest radiograph showed displacement of the heart and mediastinum to the left, elevation of the left hemidiaphragm, and thinwalled ring shadows anteriorly in the left zones consistent with emphysematous bullae.

Pulmonary function tests showed moderately severe obstructive airways disease, partially reversible with bronchodilators. Bronchography showed severe bronchiectasis of all the basal segments of the left lower lobe.

Cardiac catheterisation showed no haemodynamic abnormalities, but coronary arteriography demonstrated a very large and tortuous vessel arising from the proximal left circumflex coronary artery (Fig. 1), which passed posteriorly over the left atrium into the lower part of the left lung where numerous small vessels were seen to fill. The remaining left coronary artery and the right coronary artery were entirely normal. Left ventriculogram showed good function except for an area of hypokinesis apicoinferiorly. Pulmonary angiogram showed that the left lower lobe pulmonary artery did not opacify, but the remaining pulmonary vasculature was normal. 


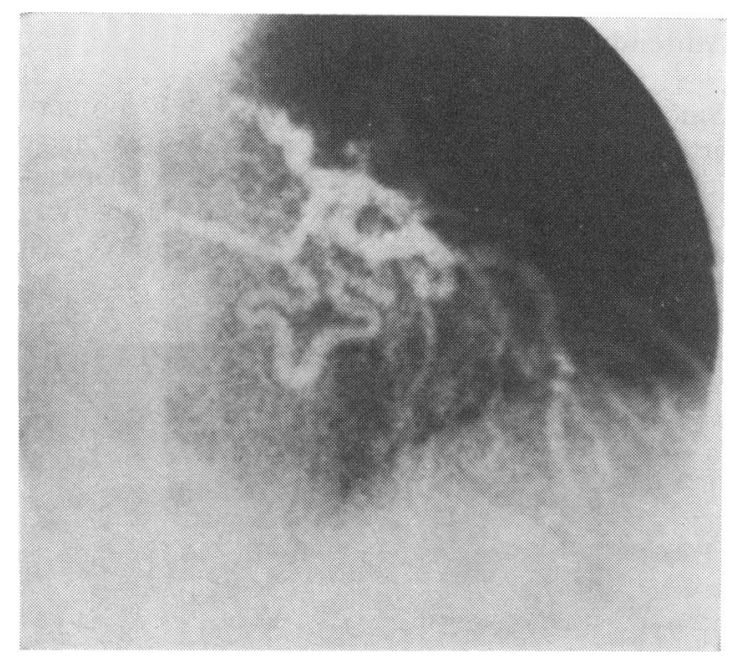

Fig. 1 Left coronary arteriogram showing filling of a large tortuous anastomotic vessel taking origin from the circumflex artery and passing posteriorly to the left lower lobe of the lung. The remaining circumflex and anterior descending coronary arteries are normal.

\section{MANAGEMENT}

Before investigation by coronary arteriography the patient's angina was treated with glyceryl trinitrate initially, but this resulted in intolerable headaches. Subsequent treatment with a series of selective beta-adrenergic blocking agents resulted in some symptomatic benefit, but exacerbation of his bronchial asthma to a degree which necessitated hospital admission and reduction in dosage. After demonstration of the coronary to bronchial artery anastomosis without coronary narrowing, it was assumed that his angina was the result of a coronary steal syndrome. As he continued to have episodes of severe chest pain, he was submitted to left thoracotomy and ligation of the anastomotic vessel, together with excision of a severely bronchiectatic left lower lobe. This resulted in complete relief of symptoms which have not recurred over a threeyear follow-up period.

\section{Discussion}

Anastomoses between the coronary arteries and bronchial arteries have been shown at necropsy ${ }^{1}$ and at cardiac catheterisation in approximately 25 per cent of normal subjects. ${ }^{2} 3$ These anastomoses are small, of little or no haemodynamic importance, and have been regarded as congenital in origin. By contrast, large anastomoses occur in pulmonary atresia, ${ }^{4}$ tetralogy of Fallot, ${ }^{5}$ supravalvar aortic stenosis, ${ }^{6}$ and coronary artery disease, ${ }^{7}$ by way of which large quantities of blood may be shunted to or from the coronary and bronchial arterial circulations. Hitherto only one case has been reported in which such an anastomosis resulted in symptoms. ${ }^{8}$ In this patient paroxysmal atrial fibrillation developed because of coronary steal from the sinuatrial artery via an anastomosis which was thought to have rendered the sinuatrial node ischaemic. ${ }^{8}$ Our patient was also symptomatic with severe angina of one year duration.

The reason for these small congenital anastomoses becoming functional is not known, but it has been suggested ${ }^{8}$ that a pressure difference between the coronary and bronchial arterial circulations may result in their enlargement and, therefore, their becoming haemodynamically important. Support for this suggestion is provided indirectly in several circumstances. Shunting of blood from the coronary to the bronchial arteries, or coronary steal, occurs either when coronary artery pressure increases relative to bronchial artery pressure as in supravalvar aortic stenosis, ${ }^{6}$ or when bronchial artery pressure decreases relative to coronary artery pressure as in pulmonary atresia, ${ }^{4}$ and tetralogy of Fallot. ${ }^{5}$ The reduction in bronchial artery pressure in these conditions is the result of filling of large capacitance, low flow, and low pressure pulmonary arteries from the bronchial arteries. Shunting of blood in the reverse direction, or bronchial steal, has been demonstrated in patients with proximal stenoses of the coronary arteries ${ }^{7}$ in whom distal coronary artery pressure is reduced compared with bronchial artery pressure. In addition, in patients with proximal coronary artery stenoses not only is the incidence of anastomoses significantly greater than normal, but a correlation has been demonstrated between the severity of coronary stenosis and the size of the anastomoses.?

Our patient had bronchiectasis, and in this condition anastomoses develop between the bronchial and the pulmonary arteries at the level of the third to sixth order bronchi, and also more distally in relation to systemic vessel proliferation in the walls of bronchiectatic sacs and destroyed lung tissue. $^{910}$ The increased bronchial arterial blood flow and large proximal bronchial to pulmonary anastomoses permit retrograde filling of the pulmonary arteries which would account for the failure of the left lower lobe pulmonary artery to opacify in our patient's pulmonary arteriogram. This lowering of bronchial arterial pressure relative to coronary arterial pressure in our patient may have led to the gross enlargement of an originally small congenital coronary to bronchial anastomosis.

Anastomoses between the coronary and bronchial arteries have similar morphology regardless of the 
direction of blood flow. ${ }^{3}$ These vessels are tortuous, of large calibre, and invariably are between the left circumflex coronary artery and the bronchial arteries, because the only point of entry to the heart is via the pericardial reflection posterior to the heart, to which the bronchial arteries are closely anatomically related. The only other possible site of coronary to bronchial anastomoses is via transpericardial vascularisation, which only occurs when the visceral and parietal pericardium are adherent after pericarditis. Coronary arteriography in our patient showed just such a large tortuous vessel taking origin from the left circumflex artery, shunting blood to the bronchial arteries. This anastomosis had presumably caused angina by a coronary steal which had deprived the inferior myocardium of blood and also resulted in the area of hypokinesis apico-inferiorly on the left ventriculogram.

\section{References}

${ }^{1}$ Von Haller A. Physiology. Troy, Ohio: Penniman, 1803: 35.

${ }^{2}$ Björk L. Anastomoses between the coronary and bronchial arteries. Acta Radiol (Diagn) (Stockh) 1966; 4: 93-6.

${ }^{3}$ Moberg A. Anastomoses between extracardiac vessels and the coronary arteries. Acta Med Scand (Suppl) 1968; 485: 5-26.

4Jefferson K, Rees S, Somerville J. Systemic arterial supply to the lungs in pulmonary atresia and its relation to pulmonary artery development. Br Heart $\mathcal{F} 1972$; 34: 418-27.

${ }^{5}$ Nordenström B. Selective catheterization and angiography of bronchial and mediastinal arteries in man. Acta Radiol (Diagn) (Stockh) 1967; 6: 13-25.

'Do Valle PV, Barcia A, Bargboun LM Jr. Angiographic study of supravalvular aortic stenosis and associated lesions. Report of five cases and review of the literature. Ann Radiol (Paris) 1969; 12: 779-96.

${ }^{2}$ Björk L. Angiographic demonstration of extracardiac anastomoses to the coronary arteries. Radiology 1966; 87: 274-7.

${ }^{8}$ Smith SC, Adams DF, Herman MV, Paulin S. Coronary-to-bronchial anastomosis. An in vivo demonstration by selective coronary arteriography. Radiology 1972; 104: 289-90.

${ }^{\circ}$ Liebow AA, Hales MR, Lindskog GE. Enlargement of the bronchial arteries and their anastomoses with the pulmonary arteries in bronchiectasis. Am f Pathol 1949; 25: 211-31.

${ }^{10}$ Turner-Warwick $M$. Pre-capillary systemic-pulmonary anastomoses. Thorax 1963; 18: 225-37.

Requests for reprints to Dr M G St John Sutton, Cardiac Department, Brompton Hospital, London SW3 6HP.

\section{Addendum}

Since describing the above patient with a coronary artery to bronchial artery anastomosis we have investigated another patient with a similar anatomical abnormality. This 44-year-old man was admitted for investigation of recurrent praecordial
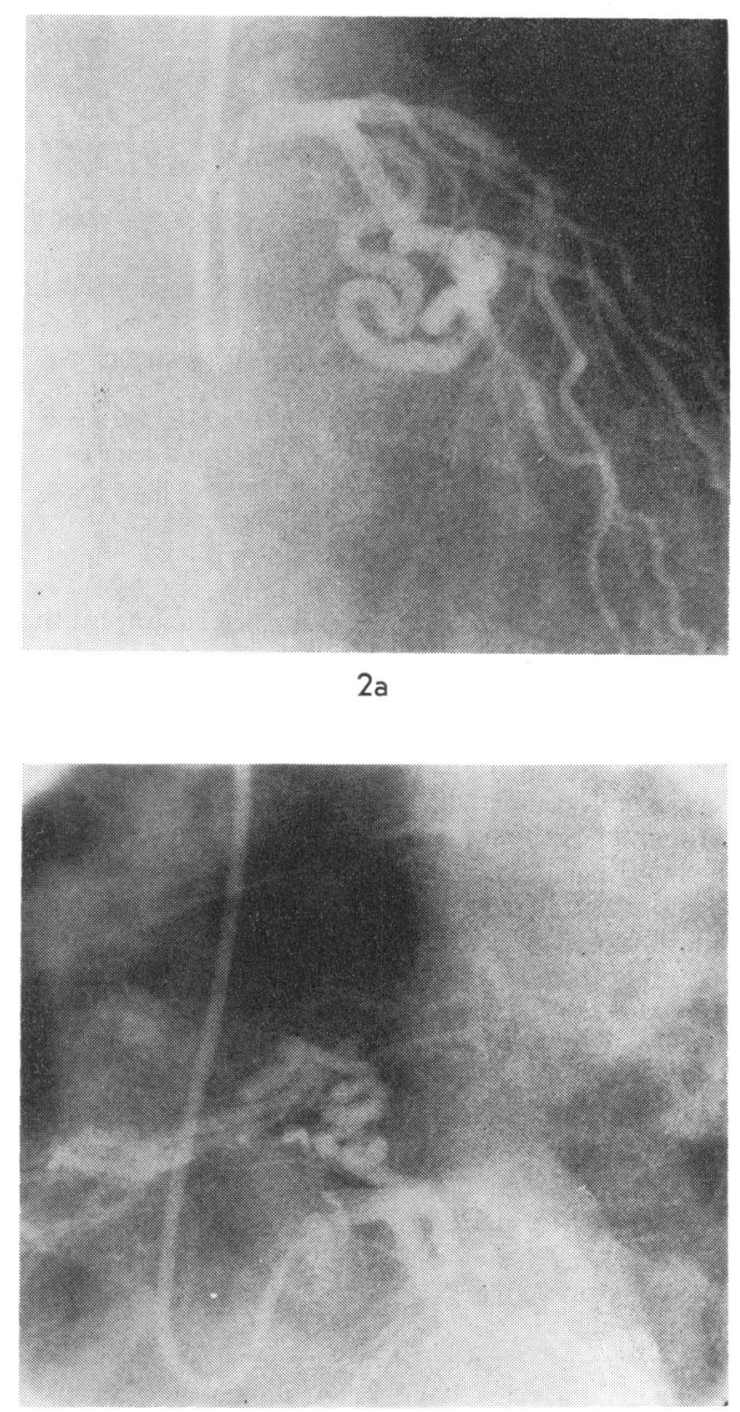

$2 b$

Fig. 2 A large tortuous artery is shown arising from the left circumflex coronary artery (a) which passes posteriorly (b) to anastomose with bronchial arteries which in turn anastomose with the right middle and lower lobe pulmonary artery (c). 
pain unrelated to exertion. Clinical examination, electrocardiogram, and chest radiograph were normal. Coronary arteriography performed to exclude coronary artery disease as a cause for the

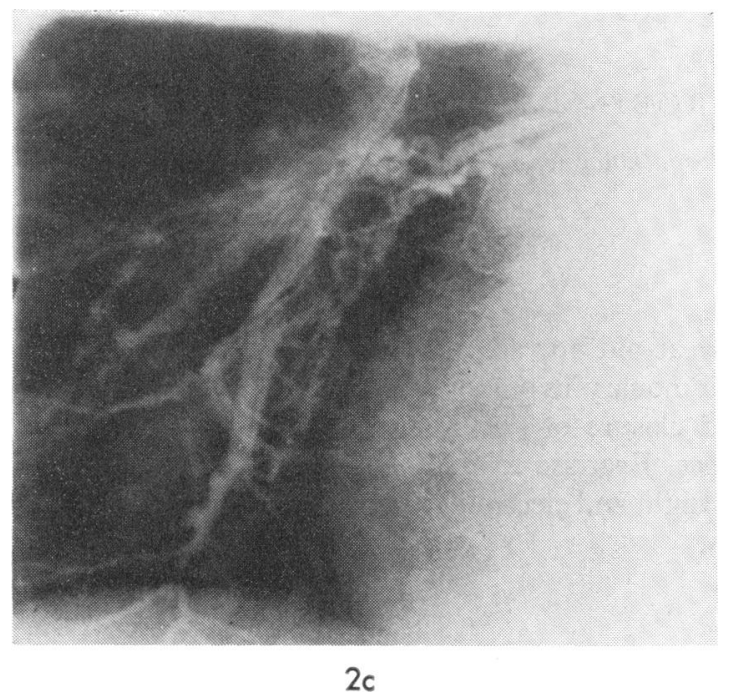

chest pain showed a very large tortuous artery arising from the left circumflex coronary artery. This vessel passed posteriorly into the mediastinum where it anastomosed with a leash of small, presumably bronchial, arteries; these in turn anastomosed with branches of the right middle and lower lobe pulmonary arteries resulting in opacification of these pulmonary arteries after the injection of contrast medium into the left coronary artery (Fig. $2 \mathrm{a} \mathrm{b}$ c). As in the previous patient there was no coronary atheroma in either the right or left coronary arteries. Because of our previous observation of the relation between coronary artery to bronchial artery fistula and bronchiectasis, bilateral bronchograms were performed. These showed moderately severe bronchiectatic changes in the right middle lobe and the apical segment of the right lower lobe. In the absence of a history of angina or severe recurrent respiratory symptoms, operation was not advised but would be reconsidered should these symptoms arise.

Our thanks are due to $\operatorname{Dr} M$ Honey for permission to publish details of this case. 